\title{
Edoxaban in dialisys: a 2-year follow-up in a single center experience
}

\begin{abstract}
Background: In literature there are not data about treatment with Edoxaban and NOACs in dialytic patients. We report our experience about.

Methods: Data for this study were retrospectively obtained from 24 consecutive patients affected by atrial fibrillation and severe chronic renal impairment in dialysis.

We analyzed the data obtained in a high-volume Italian cardiological center.

Results: All patients included were treated with Edoxaban for atrial fibrillation. At the time of the data collection, the mean follow-up in valvular patients was $24 \pm 2$ months. There were no major bleedings, strokes, transitory ischemic event, systemic embolisms, or cardiovascular deaths were been reported. 3(12,5\%) cases of minor bleeding was reported. The complication rate of the patients in dialysis are the same than the atrial fibrillation population without any statistical differences $(1,39 \%$ vs $1,37 \% \rightarrow$ p:0,96).
\end{abstract}

Conclusion: The use of Edoxaban in dialysis and atrial fibrillation seems safe and effective.

Keywords: renal impairment, dialysis, edoxaban, atrial fibrillation, safety, efficacy, complication rate
Volume I4 Issue 2 - 202I

\section{Giovanni Fazio, Leonardo Ingrasciotta, Gaspare Milana \\ Deaprtment of cardiology, Triolo Zancla Hospital, Italy}

Correspondence: Giovanni Fazio, Deaprtment of cardiology, Triolo Zancla Hospital,Via Albiri 3/a - 90125 - Palermo, Italy, Tel +39.3334439962, Email Faziogiova@gmail.com

Received: April 03, 2021 | Published: April 22, 2021

\section{Introduction}

European and American guidelines advise the use of new anticoagulation therapy in patients affected by atrial fibrillation, and in clinical practice many cardiologists us these drugs currently. The Italian drug agency authorizes the prescription in dialytic patients, even if in literature there are few inconclusive studies, all with Apixaban.

From 2012 to date, numerous revisions of the European guidelines on atrial fibrillation have been published, a progressive enlargement of treatment with NOACs in patients with a progressive low clearance. ${ }^{1-6}$ Edoxaban has been tested till now in patients with a clearance of creatinine higher than 15. It is uncleared if the ultrafiltration is able to reduce the hematic concentration of Edoxaban. In our experience, we have carried out a retrospective observational study on a cohort of 24 patients suffering from atrial fibrillation, treated with Edoxaban and with renal impairment. They did dialysis before the medical visit.

\section{Methods}

Data for this present study was retrospectively obtained from consecutive patients suffering from $\mathrm{AF}$ in one high-volume Italian cardiological centers by analyzing the reports of patients with NVAF who were included in the Italian Registry of Drugs (AIFA registry). Selected patients received a prescription for Edoxaban June 2016 and another one in September 2019. In order to be included in this study, patients had to meet these criteria: at least one episode of documented $\mathrm{AF}$ of any duration in the previous 12 months, a $\mathrm{CHA}_{2} \mathrm{DS}_{2}$-VASc [Congestive heart failure, Hypertension, Age $\geq 75$ years, Diabetes mellitus, previous Stroke/transient ischemic attack [double weight]Vascular disease, Age 65-74years old, (female) Sex category] score $\geq 2$; any type of NVAF; age $>18$ years; and a bio-prosthetic aortic valve implantation before the Edoxaban administration. All data was compared to a data obtained from the AIFA Registry of all 2251 patients affected by atrial fibrillation of our institution. To compare the results on the valvar patients, we computed the percentage incidence of events. All procedures performed in studies involving human participants were in accordance with the ethical standards of the institutional and/or national research committee and with the 1964 Helsinki Declaration. Informed consent was obtained from all individual participants included in the study, who consented to the analysis and storage of their data in the AIFA registry. Due to the retrospective nature of the study, the ethical committees were retrospectively informed of the present study. Standard twodimensional transthoracic echocardiographic examination was always performed. Left ventricular end-diastolic volume, endsystolic volume, and ejection fraction (LVEF) were measured using the modified Simpson's rule from the apical view. Follow-up, characterized by clinical examination, Electrocardiogram and blood analysis, was performed every 6 months. None of the 36 patients was lost in follow-up.

The main endpoint was the incidence of major bleedings according to the International Society on Thrombosis and Hemostasis definition, or clinically relevant non-major (CRNM) bleedings or thromboembolic events, ischemic or hemorrhagic stroke, systemic embolism, and cardiovascular death. A secondary safety endpoint was total minor bleedings. All patients' follow-up data was blinded and analyzed with the SPSS ${ }^{\circledR}$ Statistics 24.0 (IBM, Armonk, NY, USA) software. Patients were analyzed for occurrence of events and for main clinical and laboratory characteristics as well as concomitant medications.

\section{Results}

The main clinical and laboratory characteristics, as well as concomitant medications for the 24 patients in the study are shown in Table 1. 
a. All patients were treated with Edoxaban for atrial fibrillation.

b. At the time of the data collection, the average follow-up in dialytic patients was $24 \pm 4$ months. There were no major bleedings, strokes, transitory ischemic event, systemic embolisms, or cardiovascular deaths reported. 3 (12,5\%) cases of minor bleeding were reported. c. The results and the comparison with all atrial fibrillation patients are reported in Table 2.

d. The complication rate of the patients in dialysis is similar to that of fibrillation patients, indeed no statistical differences were found.

Table I Concomitant medications for the 24 patients

\begin{tabular}{|c|c|c|c|}
\hline & Dialysis & All patients & \\
\hline No. of patients $[n(\%)]$ & 24 & 2251 & \\
\hline Age (years) [mean $\pm S D]$ & $73,7 \pm 6.9$ & $76,4 \pm 2.4$ & NS \\
\hline Male/female $[n(\%)]$ & $44 \% / 56 \%$ & $52 \% / 48 \%$ & NS \\
\hline Creatinine $(\mathrm{mg} / \mathrm{dL})[$ mean $\pm \mathrm{SD}]$ & $3.86 \pm 1.2$ & $0.96 \pm 0.7$ & NS \\
\hline Months of anticoagulation therapy (mean \pm SD) & $24 \pm 2$ & $37 \pm 15$ & NS \\
\hline Paroximal o Persistent AF [n (\%)] & $88,50 \%$ & $18,50 \%$ & NS \\
\hline Permanent AF [ $n(\%)]$ & $12,50 \%$ & $72,35 \%$ & NS \\
\hline Hypertension [n (\%)] & $79 \%$ & $83 \%$ & NS \\
\hline Hypertensive Cardiomyopathy & $16 \%$ & $50 \%$ & NS \\
\hline Diabetes mellitus [ $n(\%)]$ & $25 \%$ & $25 \%$ & NS \\
\hline Heart failure $[n(\%)]$ & $8,30 \%$ & $7,40 \%$ & NS \\
\hline Previous stroke/TIA [ $n(\%)]$ & $8,30 \%$ & $8,25 \%$ & NS \\
\hline Ischemic cardiomyopathy [n (\%)] & $10,25 \%$ & $9,25 \%$ & NS \\
\hline$\beta$-Blockers $[n(\%)]$ & $36,25 \%$ & $48,25 \%$ & NS \\
\hline ACE inhibitors or ARB blockers [n (\%)] & $66,00 \%$ & $61,00 \%$ & NS \\
\hline Digossin [n (\%)] & $25,00 \%$ & $55,75 \%$ & NS \\
\hline RAAS inhibitors [n (\%)] & $8,30 \%$ & $30,00 \%$ & NS \\
\hline Amiodaron [n (\%)] & $16,00 \%$ & $13,00 \%$ & NS \\
\hline Neprilisine Inhibitor [n (\%)] & $8,30 \%$ & $6,95 \%$ & NS \\
\hline LVEF (mean $\pm S D)$ & $58 \%$ & $56 \%$ & NS \\
\hline Left atrium diameter $(\mathrm{mm})[$ mean $\pm S D]$ & $19.0 \pm 3.1$ & $22.2 \pm 3.1$ & $<0.001$ \\
\hline CHA2DS2-VASc score (mean $\pm S D)$ & $4.02 \pm 1.5$ & $4.12 \pm 2.5$ & NS \\
\hline HAS-BLED score (mean \pm SD) & $2.50 \pm 0.7$ & $2.08 \pm 1.1$ & NS \\
\hline
\end{tabular}

Table 2 The comparison with all atrial fibrillation patients

\begin{tabular}{llll}
\hline & Dialysis & All patients & P \\
\hline Major bleadings & $0 \%$ & $0,004 \%$ & NS \\
CRNM & $0 \%$ & $0,02 \%$ & NS \\
Cardiovascular death & $0 \%$ & $0,004 \%$ & NS \\
Minor bleadings & $12,50 \%$ & $11,2 \%$ & NS \\
TIA & $0,00 \%$ & $1,1 \%$ & NS \\
Stroke & $0 \%$ & $0,02 \%$ & NS \\
Systemic embolism & $0 \%$ & $0,001 \%$ & NS \\
Pulmonary embolism & $0 \%$ & $0,001 \%$ & NS \\
Cardiovascular death & $0 \%$ & $0,004 \%$ & NS \\
Complication rate & $1,39 \%$ & $1,37 \%$ & NS
\end{tabular}

\section{Discussion}

The rationale of the use of NOACs in patients in dialysis is based on few studies and until now no clinical trial nor collection in human were reported. ${ }^{1-6}$

The high risk of thromboembolic events and the same time the high risk of bleeding in this class of patients have been demonstrated.

The use of warfarin in this class of patients is the best opportunity today, but with a non-negligible risk of kidney warfarin' disease. Due to a major activation of the prothrombin factor in patients in dialysis, the thromboembolic risk is higher than in population without a renal impairment. Few studies with apixaban seem to demonstrate the safety of factor $\mathrm{X}$ inhibitor in treatment of these patients. However, the elimination of the drugs in patients in dialysis depending on the ultrafiltration so the results of apixaban cannot enlarge to other factor X inhibitors. Until now the use of Edoxaban has been tested in patients with a clearance $>15$. An in vitro study showed that dialysis is 
able to eliminate this drug from blood. In order to answer the question, we analyzed the data of patients affected by atrial fibrillation who performed dialysis.

We followed 24 patients in dialysis who were treated with Edoxaban in all cases due to the presence of atrial fibrillation. In order to evaluate the efficiency of Edoxaban in this subpopulation, we compared the data relative to safety and effectiveness events to the general ones of all fibrillating patients followed at our institution. The comparison demonstrated the efficiency and safety of an Edoxaban approach in this subpopulation, with an overall event index similar in the two populations.

The limited number of patients is the principal limitation of the study, as it prevented any sub-analysis of the type of events.

It is interesting to note that in this population the characteristic of the heart is normal in many patients, and the atrial fibrillation could be due to electrolytic disorders of these patients: in this population the atrial fibrillation is frequently paroxysmal and seriate holter monitoring may be registered in patient in dialysis.

\section{Conclusion}

In our experience, we have used Edoxaban in a population of 24 subjects in dialysis and with an atrial fibrillation: the event rate of this subpopulation was not different from that of all patients with fibrillation.

\section{Acknowledgments}

We are gratefully to Giulia Madonia and Rita Tripi for the collection of data.

\section{Conflicts of interest}

The rest of the authors declare do not have conflicts of interest.

\section{Funding}

No financial support.

\section{References}

1. Conen D, Chae CU, Glynn RJ, et al. Risk of death and cardiovascular events in initially healthy women with new-onset atrial fibrillation. JAMA. 2011;305(20):2080-2087.

2. Lip HYH, Collet JP, Caterina R, et al. Antithrombotic therapy in atrial fibrillation associated with valvular heart disease: a joint consensus document from the European Heart Rhythm Association (EHRA) and European Society of Cardiology Working Group on Thrombosis, endorsed by the ESC Working Group on Valvular Heart Disease, Cardiac Arrhythmia Society of Southern Africa (CASSA), Heart Rhythm Society (HRS), Asia Pacific Heart Rhythm Society (APHRS), South African Heart (SA Heart) Association and Sociedad Latinoamericana de Estimulación Cardíaca y Electrofisiología (SOLEACE). Europace. 2017;19(11):17571758.

3. Carnicelli A, De Caterina R, Halperin J, et al. Edoxaban for the prevention of thromboembolism in patients with bioprosthetic valves. Circulation. 2016:134

4. De Caterina R, Renda G, Carnicelli AP, et al. Valvular heart disease patients on edoxaban or warfarin in the engage aF-tiMi 48 trial. $\mathrm{J} \mathrm{Am} \mathrm{Coll}$ Cardiol. 2017;69(11):1372-1382.

5. Renda G, Ricci F, Giugliano RP, et al. Non-Vitamin K antagonist Oral anticoagulants in Patients With atrial Fibrillation and Valvular heart Disease. J Am Coll Cardiol. 2017;69(11):1363-1371.

6. Malik Ah, Yandrapalli S, Aronow WS, et al. Oral anticoagulants in atrial fibrillation with valvular heart disease and bioprosthetic heart valves. Heart. 2019;105(18):1432-1436. 